\title{
Minimally Invasive Septal Myectomy for Hypertrophic Obstructive Cardiomyopathy
}

\author{
Farah N. Musharbash, BS, Matthew R. Schill, MD, Matthew C. Henn, MD, and Ralph J. \\ Damiano Jr, MD \\ Division of Cardiothoracic Surgery, Department of Surgery, Washington University School of \\ Medicine, Barnes-Jewish Hospital, St. Louis, MO USA
}

\begin{abstract}
Surgical septal myectomy is the treatment of choice for patients with symptomatic hypertrophic obstructive cardiomyopathy refractory to medications. This report describes our minimally invasive approach for performing a septal myectomy via a mini-sternotomy that has been used at our institution for over a decade. In particular, patient preparation, surgical technique, and clinical considerations are highlighted. Performed properly, this minimally invasive technique is a feasible and effective approach in our experience.
\end{abstract}

\section{Keywords}

Septal myectomy; Minimally invasive surgery; Hypertrophic obstructive cardiomyopathy

\section{Introduction}

Septal myectomy is the primary treatment choice for patients with hypertrophic obstructive cardiomyopathy (HOCM) refractory to medical management. The procedure is reproducible, safe, and provides long-term symptomatic relief and survival benefit (1). The traditional operative approach for a septal myectomy is through a full median sternotomy (2), with very limited reports of more minimally invasive approaches (3-5). In the past decade, our institution has developed a reliable minimally invasive approach via a mini-sternotomy.

\section{Technique}

Patient selection

All patients undergoing surgical septal myectomy are considered for a mini-sternotomy as this is our preferred approach. There are a few relative contraindications. First, it can be difficult in patients who have had a previous sternotomy. A preoperative CT scan is

\footnotetext{
Address correspondence and reprint requests to Ralph J. Damiano, Jr., MD, Division of Cardiothoracic Surgery, Department of Surgery, Washington University School of Medicine at Barnes-Jewish Hospital, 660 S. Euclid Ave., Campus Box 8234, St. Louis, MO USA damianor@wustl.edu.

Disclosures: Ralph J. Damiano, Jr., MD, is a consultant for and receives research funding from AtriCure, Inc., West Chester, OH USA, and has received speakers' fees from LivaNova, London, UK. Farah N. Musharbash, Matthew R. Schill MD, and Matthew C. Henn MD declare no conflicts of interest.
} 
performed in these patients to determine the safety and feasibility. In a similar fashion, patients with a significant leftward deviation of the aorta visible on a chest $\mathrm{x}$-ray, a screening chest $\mathrm{CT}$ scan is performed to determine patient suitability.

Finally, in patients undergoing concomitant surgery that requires a wider exposure such as CABG, mitral valve repair or replacement, and/or the Cox-Maze procedure for atrial fibrillation, a sternotomy is required.

\section{Preparation}

All patients undergo a standardized preoperative evaluation including transthoracic echocardiography, coronary angiography, and anesthesia assessment. Transthoracic stress echocardiography is used to obtain resting peak left ventricular outflow tract (LVOT) gradient, septal and left ventricle wall thickness, left atrial diameter, and to check for mitral insufficiency and systolic anterior motion of the mitral valve (SAM). Provocable LVOT gradients are obtained through exercise, amyl nitrite injection, or Valsalva maneuver. All patients undergo coronary angiography to identify any existing coronary artery disease.

In the operating room, patients are placed in supine position and general anesthesia is induced. All patients undergo intraoperative transesophageal echocardiography (TEE) to confirm preoperative findings and to aid in planning the extent of the myectomy. This includes measuring the maximal depth of the septum at its thickest point as well as the extent of the septal bulge and its' distance from the aortic annulus. The skin is marked from the second to the fourth intercostal spaces in the midline (Figure 1), and the patient is prepped and draped in the usual sterile fashion. After the skin incision, a mini-sternotomy is performed from the sternal notch to the fourth intercostal space. The sternal edges are retracted, and the heart is suspended in a pericardial cradle.

\section{Cardiopulmonary bypass and cardioplegia}

After full heparinization, cardiopulmonary bypass is established in the standard fashion by direct cannulation of the ascending aorta and right atrial appendage (RAA). A flat three stage venous cannula is used for the RAA (Medtronic, Inc., Minneapolis, MN USA) and an 8 Fr. flexible cannula for the aorta (Edwards Lifesciences Corp, Irvine, CA USA). A Blake drain is placed percutaneously via a stab incision below the sternum and positioned into the posterior pericardial space for carbon dioxide infusion to prevent air embolism. A thermistor is inserted into the ventricular septum to measure myocardial temperature. The aortic root is fully mobilized, and an aortic needle vent is placed in the proximal ascending aorta for the administration of antegrade cardioplegia. The aorta is then cross-clamped and the heart is arrested with cold blood cardioplegia. A single antegrade dose is all that is usually required. If there is need for an extended period of arrest, subsequent cardioplegia is delivered by direct coronary ostia infusion. After cardioplegia is delivered, we remove the cardioplegia cannula in order to improve exposure.

\section{Myectomy (Supplemental Video)}

The aorta is opened transversely and 4-0 polypropylene stay sutures are placed to expose the aortic valve. The valve leaflets are inspected for any abnormalities (Figure 2A). The 
ventricular septum is exposed by retracting the right cusp of the aortic valve using a flexible arm retractor with custom-designed retractor blade fixed onto the sternal retraction system (AtriCure, Inc., West Chester, OH USA). If the septum cannot be adequately visualized due to leftward deviation, then we convert to a full sternotomy at this point. This occurs in less than $5 \%$ of patients from our experience. A ribbon retractor is then placed through the aortic valve and used to retract and protect the anterior mitral leaflet and the subvalvular mitral apparatus from injury (Figure 2B). The ribbon retractor is bent 90 degrees at its distal extent to prevent inadvertent injury to the papillary muscles or left ventricle. Using a \#15 blade scalpel, a septal myectomy is performed beginning 1-2 $\mathrm{mm}$ below the aortic leaflet insertion and extending from the nadir of the right cusp of the aortic valve leftward to the commissure between the left and right cusps. The myectomy is extended down towards the edge of the anterior leaflet of the mitral valve, and carried inferiorly as far as needed to remove all of the obstructive muscle. This is usually carried down to the mid-papillary muscle level, but can be deeper as defined by the intraoperative TEE. Adjusting the flexible arm retractor deeper into the myectomy trough can improve visibility as the myectomy is extended inferiorly. Importantly, the use of long endoscopic, single-shafted forceps and scissors facilitate the exposure for the myectomy. It is worth mentioning that the incision should not begin further to the right than the nadir of the right coronary cusp, due to the risk of injuring the membranous septum or the conduction system.

Following the myectomy, the outflow tract is inspected for accessory papillary muscles and chords, which when present are excised as they can contribute to the obstruction. In patients with markedly hypertrophied secondary chords, it has been our policy to excise them if they appear to crowd the outflow tract. Finally, all visible endocardial scar is removed. The aortic and mitral valves are then inspected to ensure they have not been injured, and the left ventricle is irrigated with iced saline. The patient is placed in steep Trendelenburg position and the aorta is closed with a running 4-0 non-absorbable monofilament polypropylene suture. The aorta is then unclamped and the patient is fully rewarmed. The ascending aorta is continuously vented. Suture lines are carefully inspected for hemostasis, and epicardial pacing wires are placed on the right ventricle and atrium. The patient is then weaned from cardiopulmonary bypass, and TEE is utilized to assess the LVOT gradient, look for presence of SAM and mitral insufficiency, rule out a ventricular septal defect and evaluate ventricular function.

\section{Closure}

The Blake drain that was placed at the beginning of the procedure is left in the pericardial space for drainage. A second drainage tube is placed percutaneously below the sternum into the right pleural space. The patient is then weaned from cardiopulmonary bypass. Once the patient is hemodynamically stable, protamine is given to reverse the heparin. The venous and arterial cannulas are removed. The chest is then irrigated with antibiotic-containing saline solution. Once meticulous hemostasis is obtained, the pericardium is loosely approximated. The sternum is reapproximated with four heavy gauge wires. The fascia, subcutaneous tissue and skin are closed in layers and sterile dressings are applied to all wounds. 


\section{Postoperative management}

The postoperative care of patients who undergo minimally invasive septal myectomy is similar to that of patients undergoing minimally invasive aortic valve surgery for aortic stenosis. Because of their hypertrophied ventricles, it is important to maintain adequate preload to preserve ventricular filling pressures. Most patients are extubated in the operating room or soon after arrival to the intensive care unit. Early mobilization is encouraged on postoperative day one and chest tubes are removed on postoperative day two. The median hospital length of stay for the minimally invasive myectomy at our institution has been 5.5 days.

\section{Discussion}

Minimally invasive techniques have been used for multiple cardiac procedures in the past decade, including coronary revascularization and for the treatment of valvular heart disease. These techniques have involved limited access, either via a mini-sternotomy or minithoracotomy. In a recent review of mini-sternotomy for aortic valve replacement, ministernotomy showed several advantages compared to full sternotomy in terms of lower blood loss, shorter ICU length of stay, and better postoperative pulmonary function (6). This is apart from the clear cosmetic advantage and patient preference towards a less invasive technique. In addition, sternal non-union and instability is virtually never seen with a ministernotomy compared to a full sternotomy, especially in obese patients.

The use of a less invasive approach for septal myectomy is particularly important since patients are often considered for either a surgical myectomy or a catheter-based septal alcohol ablation. Both procedures have been shown to provide similar symptomatic improvement with a similar risk of procedural mortality. However, several meta-analyses have shown that septal alcohol ablation is associated with a greater risk of some complications such as atrioventricular block and need for a permanent pacemaker (7). Therefore providing the option to the patient of a minimally invasive surgical approach would be helpful in terms of the shared-decision making process, particularly considering that a surgical myectomy has broader indications, better long-term outcomes, and fewer conduction complications compared to septal alcohol ablation. The challenge to this approach is mostly associated with the deviation of the septum in certain patients. However, a mini-sternotomy can be more easily converted to a full sternotomy if needed.

In our experience, minimally invasive septal myectomy is a feasible and easy-to-perform approach for patients with hypertrophic obstructive cardiomyopathy refractory to medications.

\section{Supplementary Material}

Refer to Web version on PubMed Central for supplementary material. 


\section{Acknowledgments}

Farah N. Musharbash, BS, is supported by the Washington University Summer Research Program Dean's Fellowship. Matthew R. Schill, MD, is supported by T32-HL007776. Ralph J. Damiano, Jr. is supported by R01HL032257.

\section{References}

1. Ommen SR, Maron BJ, Olivotto I, et al. Long-term effects of surgical septal myectomy on survival in patients with obstructive hypertrophic cardiomyopathy. J Am Coll Cardiol. 2005; 46:470-476. [PubMed: 16053960]

2. Schaff HV, Said SM. Transaortic extended septal myectomy for hypertrophic cardiomyopathy. Oper Tech Thorac Cardiovasc Surg. 2012; 17:238-250.

3. Gilmanov D, Bevilacqua S, Solinas M, et al. Minimally invasive septal myectomy for the treatment of hypertrophic obstructive cardiomyopathy and intrinsic mitral valve disease. Innovations. 2015; 10:106-113. [PubMed: 25803770]

4. Khalpey Z, Korovin L, Chitwood WR Jr, et al. Robot-assisted septal myectomy for hypertrophic cardiomyopathy with left ventricular outflow tract obstruction. J Thorac Cardiovasc Surg. 2014; 147:1708-1709. [PubMed: 24793596]

5. Mazine A, Ghoneim A, Bouhout I, et al. A novel minimally invasive approach for surgical septal myectomy. Can J Cardiol. 2016; 32:1340-1347. [PubMed: 27062240]

6. Kirmani BH, Jones SG, Malaisrie SC, Chung DA, Williams RJ. Limited versus full sternotomy for aortic valve replacement. The Cochrane Library. 2017

7. Elliott PM, Anastasakis A, et al. Authors/Task Force m. 2014 ESC Guidelines on diagnosis and management of hypertrophic cardiomyopathy: The Task Force for the Diagnosis and Management of Hypertrophic Cardiomyopathy of the European Society of Cardiology (ESC). Eur Heart J. 2014; 35:2733-2779. [PubMed: 25173338] 


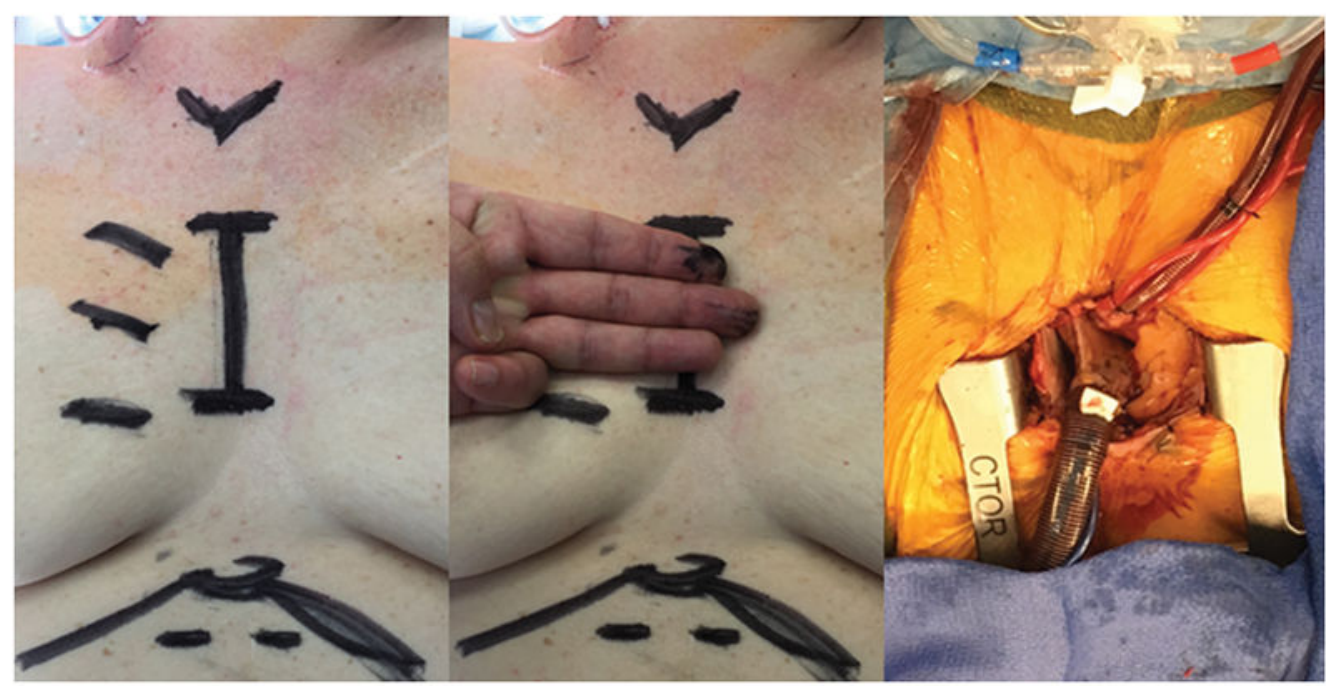

Figure 1.

Photograph showing the mini-sternotomy incision, which extends from the second to the fourth intercostal space. 

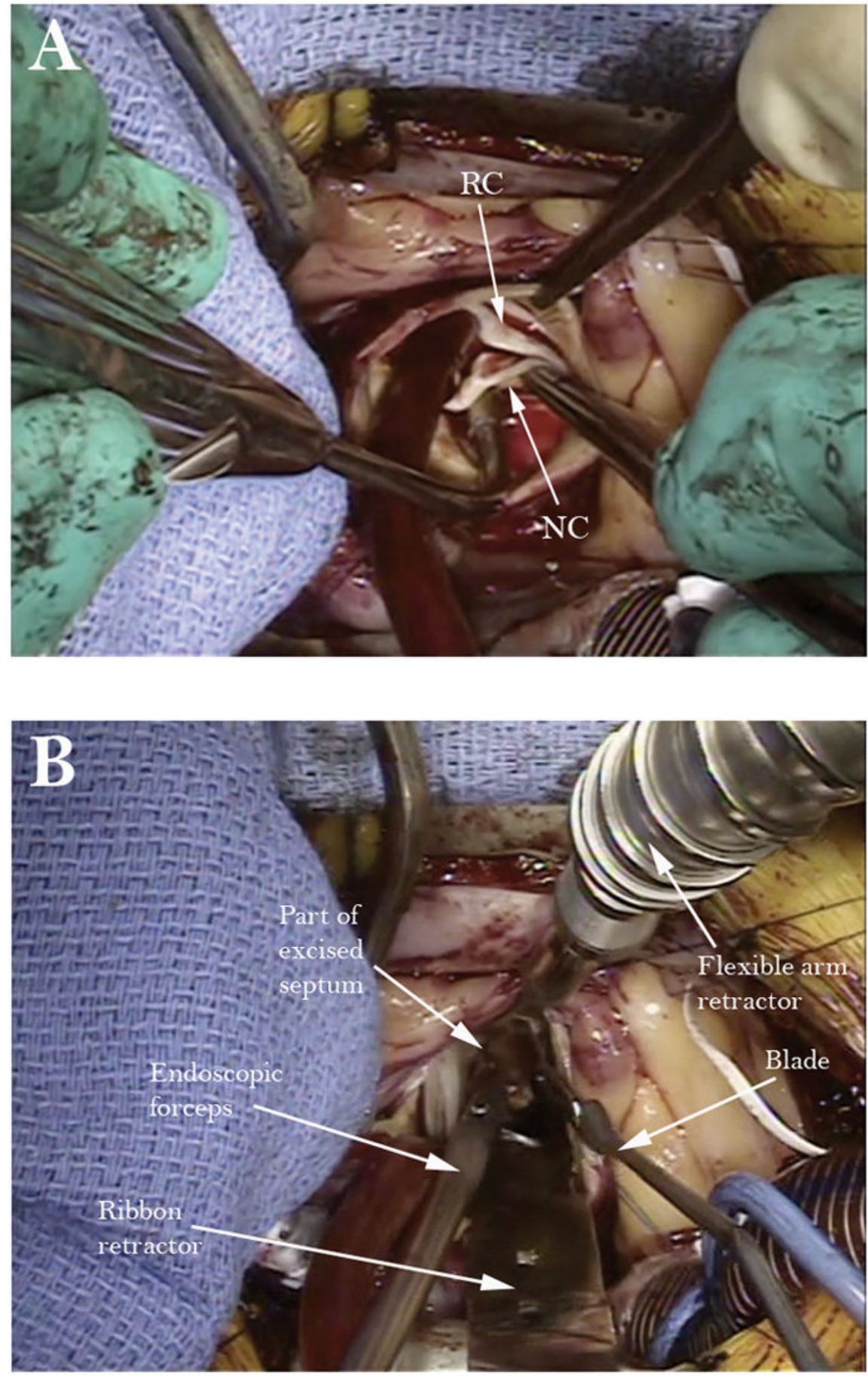

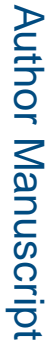




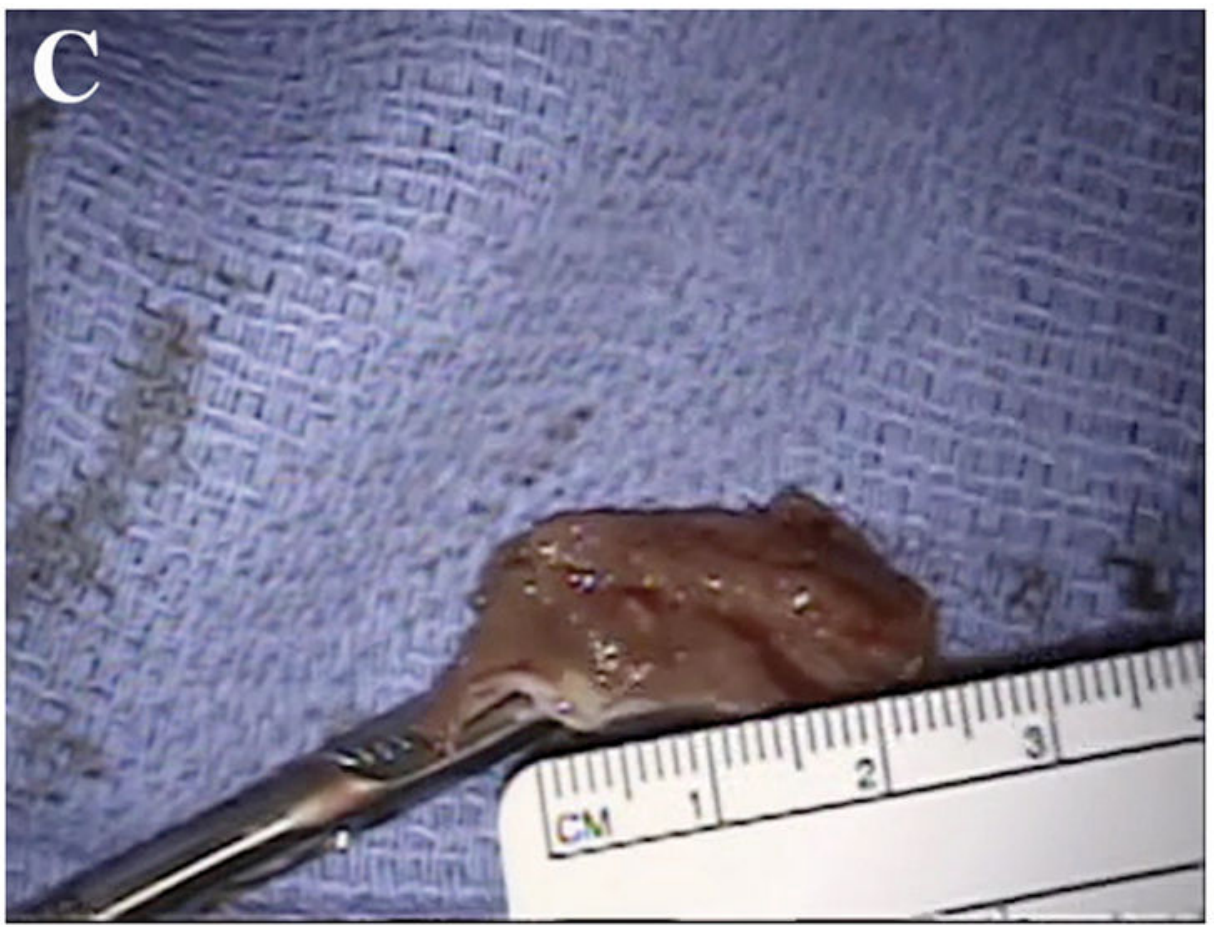

Figure 2.

A) Right cusp (RC) and non-coronary cusp (NC) of the aortic valve. B) Setup of instruments during the myectomy. C) Initial excised part of the hypertrophied septum; the excision is then extended further as needed. 